\title{
Individual pitch functions and pitch-duration cross-dimensional matching
}

\author{
STEPHEN W. PAINTON, WALTER L. CULLINAN, and EUGENE O. MENCKe \\ University of Oklahoma Health Sciences Center, Oklahoma City, Oklahoma 73190
}

\begin{abstract}
Eight young adult subjects scaled the auditory dimensions of duration and pitch using a modified method of numerical magnitude balance and adjustment of the stimuli for individual equal-loudness differences. Individual duration and pitch functions for magnitude estimation and for magnitude production fit the power law well. When compared with the revised mel scale and the pitch function obtained by S. S. Stevens and Galanter (Journal of Experimental Psychology, 1957, 54, 377-411), the group magnitude estimation and magnitude production pitch functions plotted in log-log coordinates showed high degrees of linearity. This was due mostly to the absence of a rollover in the high-frequency range of the continuum. It was hypothesized that pitch may be viewed as a linear function. This hypothesis was further supported when the exponents of the pitch and duration functions were used to predict closely the group exponent of cross-dimension matches.
\end{abstract}

Three continua of interest to psychoacousticians are duration, loudness, and pitch. Duration and loudness are viewed extensively as being prothetic continua and have been shown in numerous psychophysical scaling studies to yield power functions (Steiner, 1968; J. C. Stevens \& Guirao, 1964; S. S. Stevens, 1956; S. S. Stevens \& Galanter, 1957). Although S. S. Stevens (1957) and S. S. Stevens and Galanter (1957) speculated that pitch might be a hybrid mixture of both prothetic and metathetic continua, pitch generally has been viewed as a metathetic continuum and is reported to be curvilinearly rather than linearly related to frequency (Beck \& Shaw, 1962; S. S. Stevens, 1958; S. S. Stevens \& Galanter, 1957). Siegel's (1965) work is one exception to this general finding. Although Siegel reported pitch to be exponentially related to frequency, his data were not displayed in $\log$-log coordinates. The pitch function, however, when plotted in log-log form does appear to be linear and does not show the lowering or leveling off of perceived pitch at higher frequencies reported in other studies (S. S. Stevens \& Galanter, 1957; S. S. Stevens \& Volkman, 1940). Siegel attributed this finding to the fact that he used a test chamber that was less acoustically absorbent for high-frequency tones than did S. S. Stevens and that, moreover, his subjects, in contrast to those of S. S. Stevens and Volkman (1940), were all under 25 years of age.

It is generally accepted that pitch data fail to yield a power function. Although data obtained over the span of a century have indicated a cross-correlation

Requests for reprints should be sent to Stephen Painton, Speech and Hearing Center, Northeastern Oklahoma State University, Tahlequah, Oklahoma 74464. between auditory pitch and visual brightness (Marks, 1974), pitch typically has been excluded from validation studies using the cross-modality or crossdimensional matching procedure. Abbey (1962) performed an experiment in which subjects matched numerosity and pitch. Failing to control for differences in loudness levels for various frequencies, he obtained a highly curvilinear pitch function. Despite this, Abbey reported that the results of the crossmodality match were predicted accurately by the magnitude estimates of each dimension, suggesting that subjects can perform a metathetic-prothetic match. Several experimental peculiarities make Abbey's results questionable, however, and a more carefully designed study is merited to determine the ability of subjects to match pitch to a prothetic continuum.

Many experiments have demonstrated that individuals are capable of producing data for prothetic continua which fit the power law (J. C. Stevens \& Guirao, 1964; J. C. Stevens \& Mack, 1959). A question remains, however, with regard to the capabilities of individuals to do the same for metathetic continua. Although pitch yielded a power function for the group of subjects in Siegel's study, data were not presented concerning individual subject performance.

The purpose of the present study, then, was to employ procedures in which better controls were used than in earlier studies to: (1) obtain pitch functions for individual subjects and determine whether or not these functions could be reasonably described by power functions; and (2) determine whether subjects were able to validate their individual functions by a cross-dimensional match of pitch with duration, a prothetic continuum. 


\section{METHOD}

\section{Subjects}

The subjects were eight graduate students (four males and four females) enrolled at the University of Oklahoma Health Sciences Center, Department of Communication Disorders. The mean age of the subjects was 22.7 years, ranging from 21 to 24 years. Each subject passed a hearing screening test. Female subjects participated in the study only during the 10 days following cessation of their menstrual period, as it has been reported that the perception of some aspects of auditory stimuli may be affected in an unpredictable manner during ovulation and menstruation (Stokinger, Note 1).

Prior to the scaling tasks, an equal-loudness function at 60 phons was obtained for each subject using the method of loudness balance. Individualized cams were constructed for a Békésy audiometer to provide each subject with an equal-loudness contour during the magnitude production task for pitch and during the cross-dimensional matching task when pitch was the variable dimension.

\section{Apparatus}

A two-room sound-treated suite was used, with the experimenter in the control room and the subject in the test chamber. The stimuli for all scaling tasks were produced by three audiooscillators, two on the experimenter's panel and the other within a Békésy audiometer in the subject's room. All stimuli were presented monaurally to the subject's right ear by a calibrated Telephonics TDH-39 earphone mounted in a supra-aural cushion. Subjects controlled the stimulus presentation rate with pushbutton switches mounted on a panel. The pushbutton switches activated Grason-Stadler 1200 Series analog devices which controlled the timing sequences for all stimuli throughout the experiment.

Subjects varied stimulus duration during the magnitude-production-of-duration tasks and the cross-dimensional matching tasks by rotating a smooth knob on the panel. This knob was connected to a 150,000 -ohm 10-turn continuously variable potentiometer which was substituted for the resistive network in one of the timers used to control stimulus duration. Subjects varied stimulus frequency during the magnitude-production-of-pitch tasks and the cross-dimensional matching tasks by rotating a smooth knob on the panel. This knob was connected to a steel shaft linked directly to the variable-frequency knob of the Békésy audiometer beatfrequency oscillator.

\section{Stimuli}

The physical durations of the seven stimuli presented in the magnitude-estimation-of-duration task and in the crossdimensional matching task when duration was the standard dimension were $0.5,1.0,1.5,2.0,3.0,5.0$, and $7.5 \mathrm{sec}$. Stimulus duration during the magnitude-production-of-duration task and the cross-dimensional matching task when duration was the variable dimension could be varied from 0.03 to $10 \mathrm{sec}$. Stimulus frequency and intensity were $1,000 \mathrm{~Hz}$ and $60 \mathrm{~dB} \mathrm{SPL}$, respectively.

The frequencies of the seven stimuli presented in the magnitudeestimation-of-pitch task and in the cross-dimensional matching task when pitch was the standard dimension were $200,450,850$, $1,550,3,500,5,000$, and $7,500 \mathrm{~Hz}$. Musical relationships, which Beck and Shaw (1962) report may affect the form of the pitch function, do not exist among these frequencies. Stimulus frequency during the magnitude-production-of-pitch task and the cross-dimensional matching task when pitch was the variable dimension could be varied from 50 to $10,000 \mathrm{~Hz}$. Stimulus duration was $2 \mathrm{sec}$ and loudness level 60 phons.

\section{Procedure}

Magnitude estimation and magnitude production procedures were employed to obtain subjective magnitude scales for both duration and pitch. For the first stimulus presented in the magni- tude estimation tasks, the subject was instructed to give any number which seemed appropriate to him. For subsequent stimuli, the subject was to assign numbers in proportion to his sensory impressions using the immediately preceding stimulus as a standard. Stimulus presentation was ordered so that each of the seven stimulus values was immediately preceded once by each of the other stimulus values. This balanced design was used to lessen assimilation bias effects noted by Ward (1973). It also avoids the effect of the frequency of a single standard on the slope of the function, as reported by Beck and Shaw (1963). Stimulus presentation order was identical for all subjects and for all magnitude estimation, magnitude production, and crossdimensional matching tasks.

For the magnitude production task, numerical values taken from the geometric means of each subject's magnitude estimates were presented in the previously mentioned balanced order. Subjects were instructed to adjust the magnitude of stimulus pitch or duration until it matched the magnitude of the presented numbers, using the immediately preceding number as a standard.

The cross-dimensional matching task consisted of subjects adjusting stimulus duration or stimulus frequency to match the apparent pitch or duration of the standard stimuli. The frequencies of the standard stimuli when duration was the variable dimension were identical to those presented in the magnitudeestimation-of-pitch tasks. Likewise, the durations of the standard stimuli when pitch was the variable dimension were identical to those presented in the magnitude-estimation-of-duration tasks.

As a preexperiment familiarization procedure, each subject judged the apparent length of a series of straight lines. Each subject also participated in a practice magnitude-estimation-ofpitch task before performing the actual magnitude-estimation-ofduration and -pitch tasks. Similarly, an abbreviated magnitude production practice session was conducted prior to the experimental magnitude production tasks.

\section{RESULTS AND DISCUSSION}

\section{Duration}

Duration functions for the eight subjects were obtained for each of the two scaling procedures, magnitude estimation and magnitude production, and are presented in Figure 1. Straight lines were fitted to the data points by the least squares method. The exponents for the power functions, the mean exponents and the product-moment correlation coefficients for estimating the degree of relationship between the physical and psychological magnitudes are presented in Table 1.

Inspection of Figure 1 clearly indicates, as expected, that the duration functions for each subject are linear in log-log coordinates with no systematic deviations from the regression line. The high correlation coefficients, ranging from 0.98 to 1.00 , provide further support for the assumption of linearity of the duration functions. The amount of bias in each subject's function due to regression is visible as the difference in slopes between the two regression lines. Subject 1 shows the most regression and Subject 3 the least.

Mean exponents for individual duration functions were derived using the method suggested by Indow and S. S. Stevens (1966) and S. S. Stevens and Greenbaum (1966), where the geometric mean of the exponents of the magnitude estimation and 


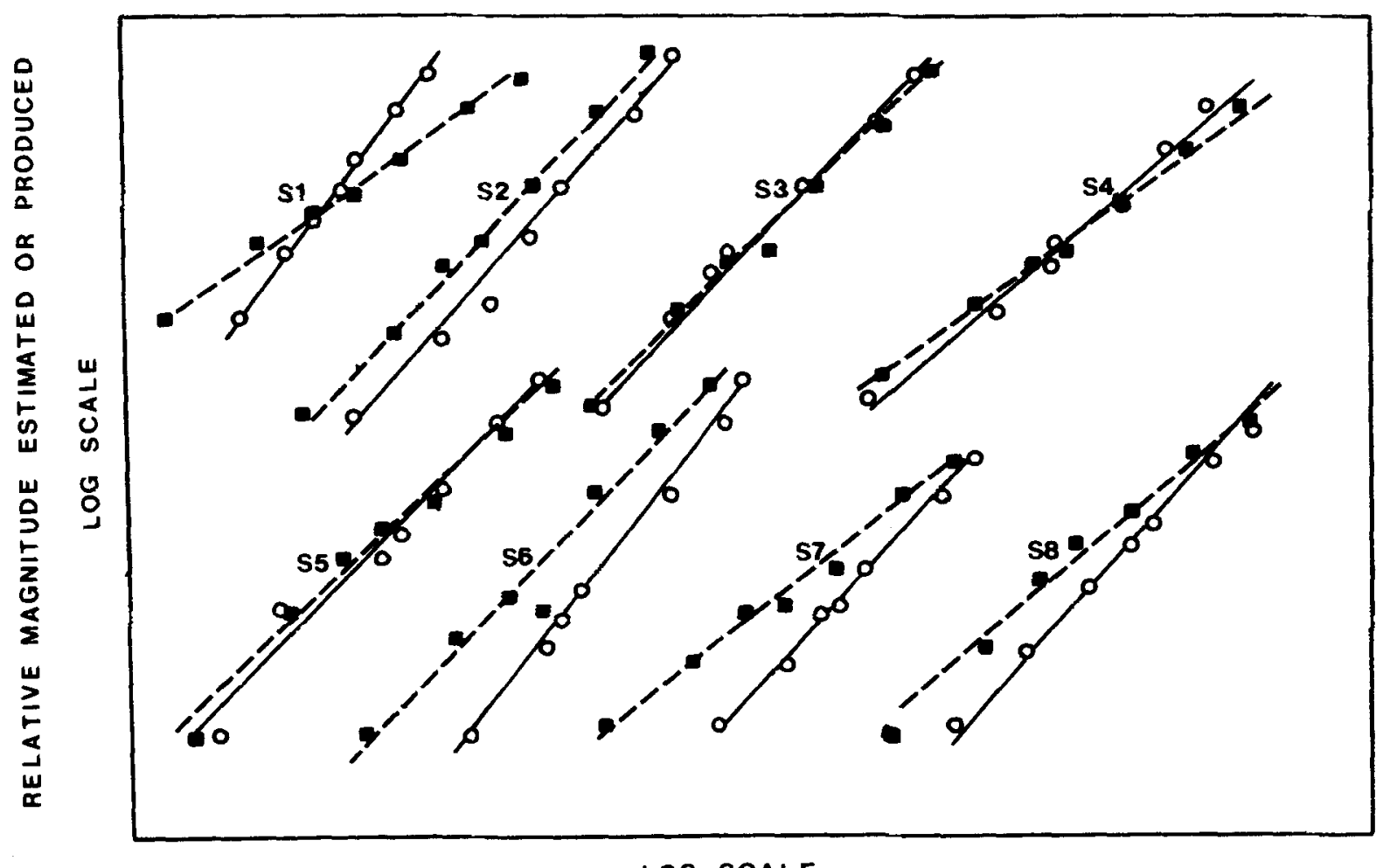

LOG SCALE

RELATIVE DURATION

Figure 1. Functions for duration obtained by the methods of magnitude estimation (solid squares and dashed lines) and magnitude production (open circles and solid lines) for each of eight subjects. The relative positions of the functions are arbitrary along both axes, but the slopes are those determined by the data.

magnitude production functions for each subject was computed. The resulting mean exponents represent the subject's duration functions presumably corrected for regression. These exponents, presented in Table 1, ranged from 0.89 to 1.13 , with the group mean of 1.02. The group geometric mean agrees closely with the duration slope of slightly greater than 1.00, as empirically determined by S. S. Stevens

Table 1

Exponents, Geometric Mean (GM) Exponents, and Correlation Coefficients Describing the Perceptual Measurement of Duration as a Function of the Physical Measure for Each Subject for Magnitude Estimation (ME) and Magnitude Production (MP)

\begin{tabular}{|c|c|c|c|c|c|}
\hline \multirow[b]{2}{*}{ Subjects } & \multicolumn{3}{|c|}{ Exponents } & \multicolumn{2}{|c|}{$\begin{array}{l}\text { Correlation Co- } \\
\text { efficients }\end{array}$} \\
\hline & $\mathrm{ME}$ & MP & GM & $\mathrm{ME}$ & MP \\
\hline 1 & .72 & 1.40 & 1.01 & 1.00 & .98 \\
\hline 2 & 1.08 & 1.15 & 1.11 & .99 & .99 \\
\hline 3 & .98 & 1.02 & 1.00 & .99 & .99 \\
\hline 4 & .82 & .97 & .89 & 1.00 & .99 \\
\hline 5 & 1.02 & 1.06 & 1.04 & .99 & .99 \\
\hline 6 & 1.03 & 1.24 & 1.13 & .99 & .99 \\
\hline 7 & .79 & 1.13 & .94 & .99 & .99 \\
\hline 8 & .94 & 1.22 & 1.07 & 99 & .99 \\
\hline GM & .91 & 1.14 & 1.02 & & \\
\hline
\end{tabular}

and others (Gregg, 1951; Ross \& Katchmar, 1951; S. S. Stevens, 1957; S. S. Stevens \& Galanter, 1957).

\section{Pitch}

Pitch functions for each subject for each of the two scaling procedures are presented in Figures 2 and 3 with straight lines fitted to the data points by the method of least squares. The exponent for the power functions, the mean exponents, and the product-moment correlation coefficients for estimating the degree of relationships between physical and psychological magnitudes are presented in Table 2.

Visual inspection of Figures 2 and 3 suggests that most subjects' magnitude estimation functions fit the power law reasonably well. An even better fit is obtained if the lowest point in the function is excluded from consideration. For Subjects 4 and 8 , in particular, the numerical estimates for the $200-\mathrm{Hz}$ stimulus, the lowest stimulus, were much lower in relation to their estimates for the other stimuli than were the estimates of the other subjects. Subjects 3 and 6 also gave estimates for the lowest point, which might be considered unusually low estimates, but the deviations are not as pronounced as those of Subjects 4 and 8 . The exclusion of the lowest points of Subjects 3 and 6 would lower the exponents 


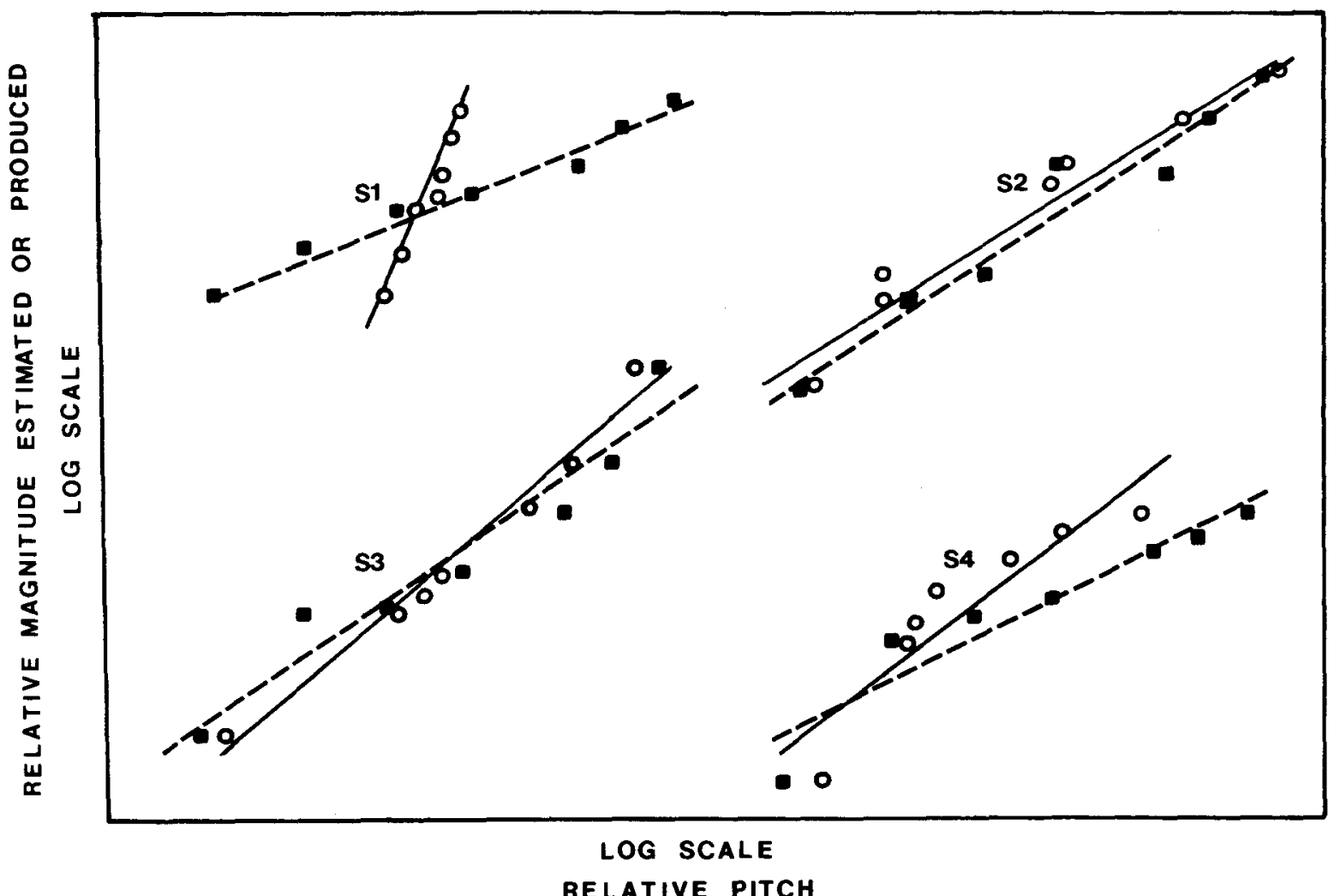

Figure 2. Functions for pitch obtained by the methods of magnitude estimation (solid squares and dashed lines) and magnitude production (open circles and solid lines) for each of four subjects (S1, S2, S3, and S4). The relative positions of the functions are arbitrary along axes but the slopes are those determined by the data.

for their functions by only 0.03 and 0.08 , respectively, below what they are with the lowest points included. The exponents for the functions of Subjects 4 and 8 , however, would be lowered by 0.14 and 0.21 , respectively, below what they are with the lowest points included.

The apparent deviation of extreme points from linearity has been observed by other investigators also. J. C. Stevens and Tulving (1957), S. S. Stevens

Table 2

Exponents, Geometric Mean (GM) Exponents, and Correlation Coefficients Describing the Perceptual Measurement of Pitch as a Function of the Physical Measure for Each Subject for Magnitude Estimation (ME) and Magnitude Production (MP)

\begin{tabular}{ccccccc} 
& \multicolumn{3}{c}{ Exponents } & & \multicolumn{2}{c}{$\begin{array}{c}\text { Correlation Co- } \\
\text { efficients }\end{array}$} \\
\cline { 2 - 3 } \cline { 6 - 7 } Subjects & ME & MP & GM & & ME & MP \\
\hline 1 & .38 & 2.56 & .99 & & .99 & .98 \\
2 & .65 & .63 & .64 & & .98 & .98 \\
3 & .69 & .87 & .78 & & .96 & .99 \\
4 & .53 & .80 & .65 & & .95 & .92 \\
5 & .39 & .55 & .46 & & .98 & .98 \\
6 & .49 & .56 & .52 & & .97 & .98 \\
7 & .96 & .87 & .91 & & .98 & .92 \\
8 & .65 & .80 & .72 & .94 & .92 \\
GM & .57 & .84 & .69 & & \\
\hline
\end{tabular}

(1955, 1956) and S. S. Stevens, Volkman, and Newman (1936), for example, described the subject's tendency to overestimate the subjective magnitude of the stimuli at the high end while underestimating it at the low end of the continuum for the dimensions of loudness and pitch. This deviation from linearity reportedly is due to the subject's consideration of the absolute values of the stimuli in addition to the relative values. Apparently, the subject is impressed with the relatively unfamiliar magnitudes of the stimuli regardless of which end of the continuum the stimuli lie on and this impression is reflected in his estimation or production. The data in the present study do not suggest as great a tendency to overestimate the stimulus with the highest frequency as to underestimate the stimulus with the lowest frequency.

Other investigators, with one notable exception (Siegel, 1965), have stated that the pitch function is not linear but rather a complex curvilinear function. In the present study, only the magnitude estimation data for Subject 3 are strongly suggestive of a curvilinear function, particularly if the lowest point is excluded. Complex curves, of course, could be fitted to the functions for each of the subjects, but the curves would differ considerably among subjects.

The question arises as to what criteria are used in 


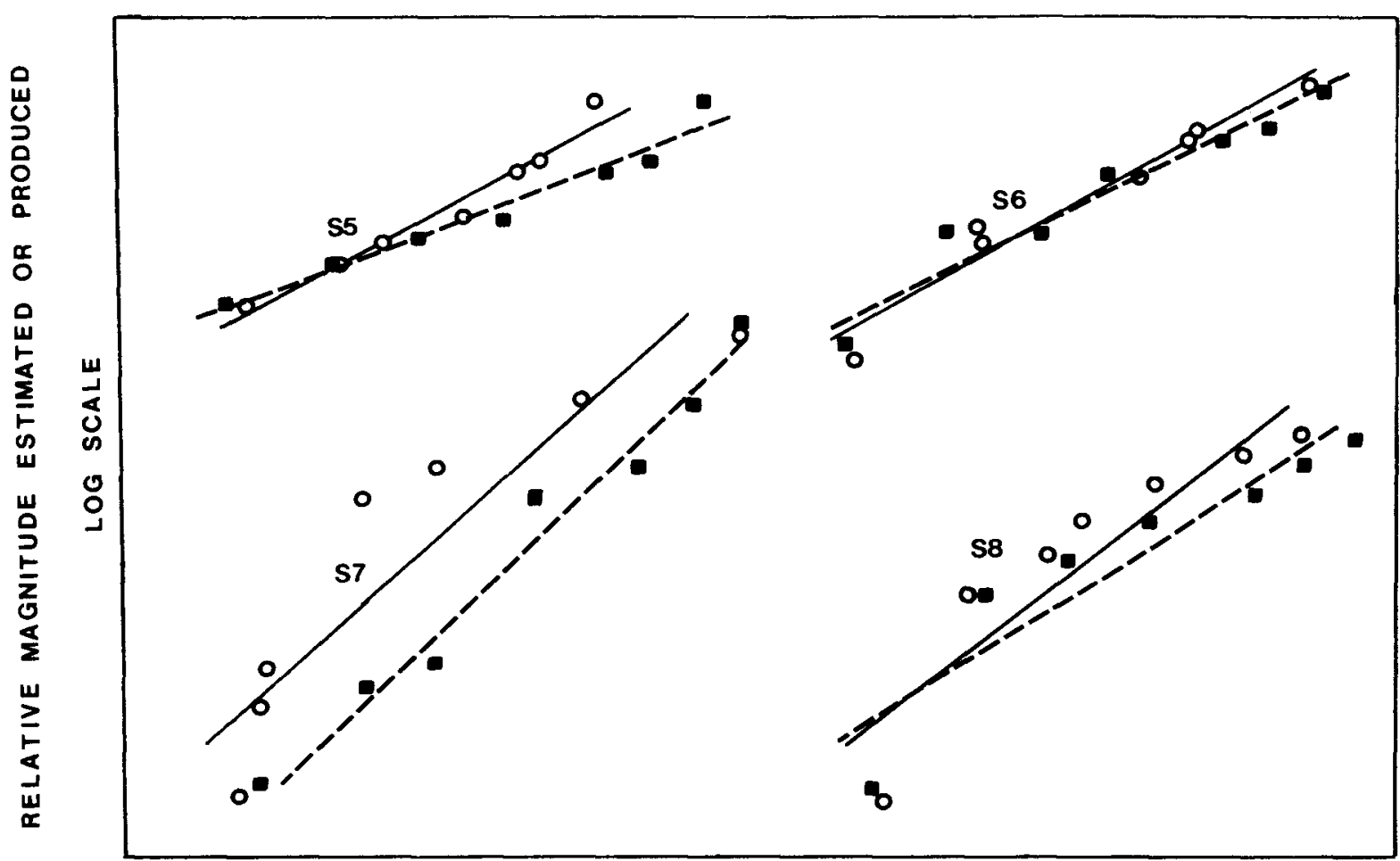

LOG SCALE

RELATIVE PITCH

Figure 3. Functions for pitch obtained by the methods of magnitude estimation (solid squares and dashed lines) and magnitude production (open circles and solid lines) for each of four subjects (S5, S6, S7, and S8). The relative positions of the functions are arbitrary along both axes, but the slopes are those determined by the data.

arriving at the decision that data may be reasonably well fitted with a straight line. In most studies of psychophysical scaling using ratio judgments, linearity has been determined solely by visual inspection of the data plots. Others have considered productmoment correlation coefficients as well. Data considered linear in some studies could equally well have been considered as complex curvilinear functions. Ward (1973), for example, stated that his data for magnitude estimation of loudness showed a "sinusoidal bowing of the points about the straight line." Nevertheless, he stated that the function was "strikingly close to a straight line," "lies on a straight line," and "nicely fit by a power function." Jones and Marcus (1961) discussed the possibility of the presence of significant curvilinearity in their magnitude estimation data for the dimensions of weight, smell, and taste. Because high product-moment correlation coefficients were obtained between the sensation magnitudes and the physical magnitudes of their stimuli, they used exponents to describe the functions. The average product-moment correlation coefficient for the Jones and Marcus data was 0.95 .

Other investigators also have assumed power function fits to their data on the basis of high productmoment correlation coefficients. J. C. Stevens and
Guirao (1964) assumed linearity when their data for the dimension of loudness had a median correlation coefficient value of 0.984 with a range from 0.685 to 0.997 . Visual inspection of their plotted data, however, suggests the presence of curvilinearity in certain individual data. Ekman, Hosman, and Lindström (1965) presented, in graphic form, individual sensation functions of roughness and smoothness. Product-moment correlation coefficients were not reported.

Using the coefficient of 0.95 as a criterion for linearity, Freides and Phillips (1966) stated that, in all but 2 of 28 cases, the power law hypothesis appeared to yield an accurate index of their empirical data. The coefficients of the functions failing to meet the criterion were 0.94 and 0.93 . S. S. Stevens (1969) fitted straight lines to two functions, one of which was admittedly concave downward and the other "noisy." Stevens hypothesized that the concave weight function may have been the result of the experimental data collection procedure utilized by Mashhour and Hosman (1968). He also stated that the "noisiness" of the data for the function of lightness of grays was no surprise because of the difficulty subjects have had in the past when scaling that dimension. According to Stevens, these func- 
tions, along with several others which were most closely fitted by straight lines, corroborated the power law "at least to a reasonable degree."

The decision regarding linearity appears to be based, therefore, not on whether data can be fitted by a curved line but on (a) whether the data closely approximate linearity as judged by visual inspection and high product-moment correlation coefficients, and (b) whether deviations from linearity are systematic in some way. Visual inspection of the plotted data and the high product-moment correlation coefficients in this study suggest that the magnitude estimation pitch data do closely approximate linearity. The median product-moment correlation coefficient for the magnitude estimation functions is 0.98 , with the coefficients ranging from 0.94 to 0.99 . Comparison of the pitch functions among subjects fails to show systematic deviations common to all subjects with the possible exception of sinusoidal bowing similar to that noted and then ignored by Ward (1973). The more pronounced curvilinear nature of the data for Subject 3 does suggest considerable variation in pitch functions among individuals.

The magnitude production functions may also be said to fit the power law reasonably well. The median product-moment correlation coefficient for the magnitude production functions is 0.98 , with the coefficients ranging from 0.92 to 0.99 . As with the magnitude estimation functions, the lowest points for Subjects 4 and 8 show the greatest deviation from a linear relationship and there is a strong suggestion of curvilinearity for the function of Subject 3. Although the data are well fitted by a straight line, there is a strong suggestion of curvilinearity also for the function of Subject 7 . In reality, the suggestion of curvilinearity for the function of Subject 7 is due to the position of the lowest three points. Apparently, this subject became confused with the unfamiliar low tones and adjusted the frequency knob to nearly identical settings for the three lowest numerical stimuli. As a result, the three data points appear on the graph in an almost vertical linear relationship. The other four data points are linearly related, with a slope approximating those of the magnitude production functions of other subjects.

The mean exponents for the individual pitch functions, presumably corrected for regression, are presented in Table 2. The range of exponents from 0.46 to 0.99 is larger than the range of exponents for duration. One possible explanation for this difference may be that pitch is a more difficult dimension to scale than is duration. Judgment of pitch is not ordinarily encountered in everyday living as often as is judgment of duration, loudness, and some other dimensions. S. S. Stevens and Galanter (1957) reported that subjects were unfamiliar with the very low and very high pitches used in their study and, as a consequence, the subjects became confused during the scaling procedure. During the present investigation, subjects constantly expressed concern with a difficulty they encountered while attempting to scale pitch, and, therefore, a certain amount of "noise" was expected in functions.

A group function for the magnitude estimation of pitch was obtained using the method suggested by S. S. Stevens (1956). The first step was to equate $1,000 \mathrm{~Hz}$ to a subjective magnitude of 1,000 for each subject's magnitude estimation function. The number 1,000 was chosen so that the group function could be compared to the revised mel scale and the pitch function of S. S. Stevens and Galanter. Because $1,000 \mathrm{~Hz}$ was not used as a stimulus value in the magnitude estimation task, its numerical subjective magnitude had to be derived by using each subject's regression formula. The number obtained for each subject was then multiplied by the appropriate factor in order to equal 1,000. All mean judgments for each subject were weighted in the same proportion. The geometric mean was obtained for all subjects' weighted judgments at each of the seven test frequencies. The seven resulting means represented the group magnitude estimation function for pitch transformed to mels. This function is presented in Figure 4 along with the revised mel scale of Stevens and Volkman (1940), the pitch function derived by Stevens and Galanter (1957), and the group magni-

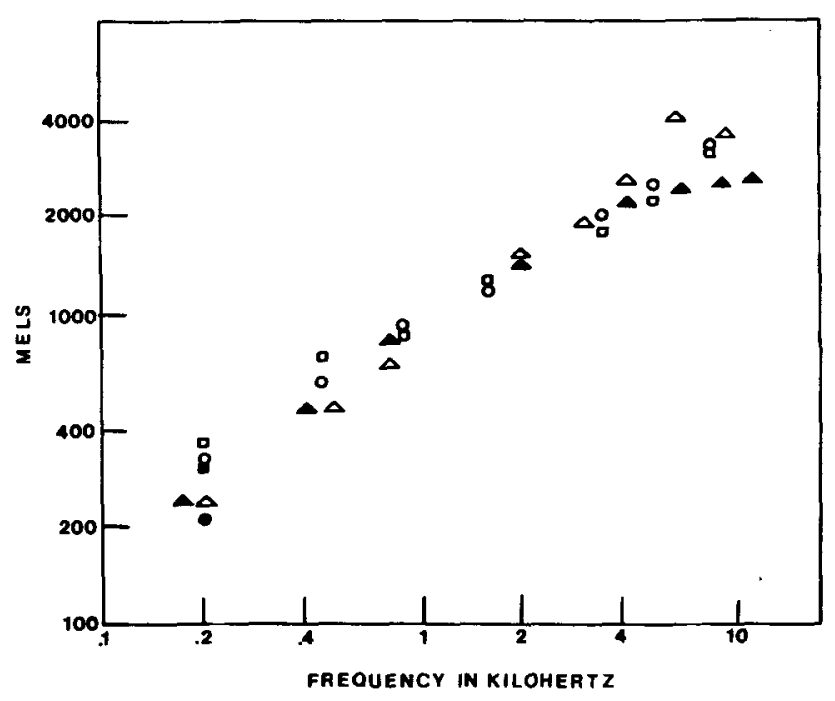

Figure 4. Comparison of the group pitch functions obtained by $S$. S. Stevens and Volkman (1940) (solid triangles), by S. S. Stevens and Galanter (1957) (open triangles), and by the present investigators, using the magnitude estimation procedure (open squares) and the magnitude production procedure (open circles). The solid square and solid circle plotted at the low end of the frequency scale represent the geometric means when the data for the two subjects which were excluded for this frequency point are included. 
tude production function from the present investigation. The lowest points on the magnitude estimation and magnitude production functions are presented both with and without the lowest points for Subjects 4 and 8 included.

The most notable difference between the magnitude estimation function obtained in this investigation and the functions of S. S. Stevens and Galanter and S. S. Stevens and Volkman is the lack, in this study, of a leveling off or a downward turn in the high frequencies. With the exception of the highest point on the frequency scale, the S. S. Stevens and Galanter data fit a linear function very well. The downward turn was very likely due to the subjects' confusion resulting from a difficulty in scaling stimuli with unfamiliar high pitches. The rollover at the upper end of the S. S. Stevens and Volkman pitch function, according to Siegel (1965), may have been due to poor acoustic characteristics of the test booth and to the loss of sensitivity to high frequencies in some subjects because of age.

It is possible, then, that the group magnitude estimation pitch function obtained in this investigation is more linear than those of the investigators mentioned previously because of improved experimental methods. The stimuli presented in the present investigation, for example, were balanced as suggested by Ward (1973) to prevent bias from assimilation. Furthermore, subjects' ages in the present study ranged from 21 to 24 years with a mean of 22.7 years. This is in contrast to Stevens and Volkman's two 34-year-old subjects. Earphones were used in the present investigation to prevent the absorption of high frequency tones into the walls. Perhaps most important, an equal loudness function was obtained for each subject in this investigation to assure minimal change in loudness throughout the pitch scaling tasks. The other investigators used group equalloudness data. It was not unusual in this investigation for individual loudness functions to differ by $10 \mathrm{~dB}$ at corresponding frequencies, especially at the low and high frequencies. In fact, differences as high as 20 to $23 \mathrm{~dB}$ were noticed at the high frequencies.

A group function for the magnitude production of pitch was obtained using a method very similar to that used by S. S. Stevens and Volkman (1940) and S. S. Stevens et al. (1936) to obtain the mel scale and the revised mel scale. The method consisted of plotting the raw magnitude production data for each subject in log-log coordinates. The points actually plotted were the geometric means of the six productions for each numerical value. Smooth curves were drawn by eye to fit the points. The values corresponding to seven frequencies used previously in obtaining the group magnitude estimation function, plus $1,000 \mathrm{~Hz}$, were extracted from the smooth curves. Each subject's function was then changed by weighting the numerical values of the subjective magnitude by the factor necessary to equate $1,000 \mathrm{~Hz}$ to a subjective magnitude of 1,000 . The individual functions were combined by calculating the geometric mean for the eight frequencies. Visual inspection of the group magnitude production function in Figure 4 strongly suggests a linear relationship. No rollover near the top of the function is noted. This, again, could be the result of the improved experimental design used in this study. The magnitude production functions of Subjects 1 and 7 were excluded from the plotted group function. The reason for excluding Subject 7 was that he produced approximately the same frequency values for the three lowest numerical values. Subject 1 was excluded because of the extreme amount of constriction shown in his magnitude production function.

\section{Cross-Dimensional Validation}

The exponents for individual obtained and predicted combined magnitude estimation and magnitude production cross-dimensional functions and the differences between them are presented in Table 3. The cross-dimensional function exponents were obtained by calculating the geometric mean of the exponents for the two cross-dimensional matching functions performed by each subject. The predicted exponents were obtained by calculating the ratios of the exponents for each subject's combined pitch function and combined duration function. The differences between the obtained and predicted exponents are small for seven of the eight subjects. Subject 7 showed a relatively large difference between his predicted and obtained exponents. It became evident after an inspection of Subject 7's data that he was unable to perform the cross-dimensional matching task. He apparently adjusted the variable dimension to the extremes of the continuum for high and low magnitudes of the standard dimension and dispersed the remaining values throughout the range without necessarily forming ratios. For this reason,

Table 3

Obtained and Predicted Exponents for Combined Cross-Dimensional Functions and Differences Between Obtained and Predicted CrossDimensional Exponents

\begin{tabular}{cccc}
\hline & \multicolumn{3}{c}{ Cross-Dimensional Exponents } \\
\cline { 2 - 4 } Subject & Obtained & Predicted & $\begin{array}{c}\text { Difference in } \\
\text { Decilogs }\end{array}$ \\
\hline 1 & .80 & .98 & .88 \\
2 & .51 & .58 & .56 \\
3 & .61 & .78 & 1.07 \\
4 & .57 & .73 & 1.07 \\
5 & .40 & .44 & .41 \\
6 & .59 & .47 & .99 \\
7 & .50 & .97 & 2.88 \\
8 & .50 & .67 & 1.27 \\
\hline
\end{tabular}


Subject 7's data are not considered in the following discussion on validation.

An investigation was conducted by Mashhour and Hosman (1968) in which 20 subjects performed magnitude estimations and cross-modality matches for seven different prothetic continua. S. S. Stevens (1969) corrected Mashhour and Hosman's data for the regression effect and presented the results in support of the power law. For the present study, the ratio of the group pitch function (0.66) and the group duration function (1.03) is 0.64 . The difference between this ratio value and the geometric mean of the subjects' cross-dimensional functions $(0.55)$ is 0.65 decilogs. This difference compares quite favorably with the range of differences given by S. S. Stevens (1969) in support of the power law. Thus, the cross-dimensional matching results appear to verify the power law for the two dimensions used in this study.

In summary, psychophysical functions for pitch obtained with the magnitude estimation and magnitude production procedures were shown to fit the power law better than has generally been reported. Carefully designed procedures and the criteria traditionally employed for making the assumption of linearity were used. The finding here held true not only for the group function but for many individual subjects as well. Subjects were able to validate their individual pitch functions by a cross-dimensional match of pitch with duration, a prothetic continuum.

\section{REFERENCE NOTE}

1. Stokinger, T. E. Personal communication, July 3, 1974.

\section{REFERENCES}

ABbeY, D. S. Cross-modality matching of numerosity and pitch. Canadian Journal of Psychology, 1962, 16, 283-290.

BECK, J., \& SHAw, W. A. Magnitude estimations of pitch. Journal of the Acoustical Society of America, 1962, 34, 92-98.

BECK, J., \& SHAW, W. A. Single estimates of pitch magnitude. Journal of Acoustical Society of America, 1963, 35, 1722-1724.

Ekman, G., Hosman, J., \& Lindström, B. Roughness, smoothness, and preference: A study of quantative relations in individual subjects. Journal of Experimental Psychology, 1965, 70, 18-26.

Freides, D., \& Phollips, P. Power law fits to magnitude estimates of groups and individuals. Psychonomic Science, 1966, 5, 367-368.
GregG, L. W. Fractionation of temporal intervals. Journal of Experimental Psychology, 1951, 42, 307-312.

Indow, T., \& Stevens, S. S. Scaling of saturation and hue. Perception \& Psychophysics, 1966, 1, 253-271.

Jones, F. N., \& Marcus, M. D. The subject effect in judgments of subjective magnitude. Journal of Experimental Psychology, 1961, 61, 40-44.

MARKs, L. E. On associations of light and sound: The mediation of brightness, pitch, and loudness. American Journal of Psychology, 1974, 87, 173-188.

Mashaour, M., \& Hosman, J. On the new "psychophysical law": A validation study. Perception \& Psychophysics, 1968, 3, 367-375.

Ross, S., \& KatchmaR, L. The construction of a magnitude function for short time intervals. American Joumal of Psychology, $1951,64,397-401$.

SIEGEL, R. J. A replication of the mel scale of pitch. American Journal of Psychology, 1965, 78, 615-620.

StEINER, S. Apparent duration of auditory stimuli. Journal of Auditory Research, 1968, 8, 195-204.

Stevens, J. C., \& Guirao, N. Individual loudness functions. Journal of the Acoustical Society of America, 1964, 36, 2210-2213.

Stevens, J. C., \& Mack. J. D. Scales of apparent force. Journal of Experimental Psychology, 1959, 58, 405-413.

Stevens, J. C., \& Tulving, E. Estimations of loudness by untrained listeners. American Journal of Psychology, 1957, 70, 600-605.

STEVENS, S. S. The measurement of loudness. Journal of the Acoustical Society of America, 1955, 27, 815-829.

STEVENS, S. S. The direct estimation of sensory magnitudes-loudness. American Joumal of Psychology, 1956, 69, 1-25.

Stevens, S. S. On the psychophysical law. Psychological Review, $1957,64,153-181$.

Stevens, S. S. Problems and methods of psychophysics. Psychological Bulletin, 1958, 55, 177-196.

STEVENS, S. S. On predicting exponents for cross-modality matches. Perception \& Psychophysics, 1969, 6, 241-256.

Stevens, S. S., \& Galanter, E. H. Ratio scales and category scales for a dozen perceptual continua. Journal of Experimental Psychology, 1957, 54, 377-411.

SteVens, S. S., \& Greenbaum, H. B. Regression effect in psychophysical judgments. Perception \& Psychophysics, 1966, 1, 439-446.

Stevens, S. S., \& Volkman, J. The relation of pitch to frequency: A revised scale. American Journal of Psychology, 1940, 53, 329-353.

Stevens, S. S., Volkman, J., \& Newman, E. B. A scale for the measurement of the psychological magnitude-pitch. Journal of the Acoustical Society of A merica, 1936, 8, 185-190.

W ARD, L. E. Repeated magnitude estimations with a variable standard: Sequential effects and other properties. Perception \& Psychophysics, 1973, 13, 193-200.

(Received for publication November 12, 1976; revision accepted March 10,1977.) 